Nunt. Antiquus, Belo Horizonte, v. 14, n. 2, p. 33-60, 2018

\title{
Les philosophes à propos du mariage : un aspect de la pensée économique antique à l'époque classique
}

\author{
Philosophers on Marriage: \\ An Aspect of Ancient Classical Greek Thought on Economics
}

\author{
Étienne Helmer \\ Université de Porto Rico, San Juan / Porto Rico \\ etiennehelmer@hotmail.fr
}

Résumé : Quel regard les philosophes grecs classiques portaient-ils sur l'économie domestique, en particulier sur la relation conjugale au sein de l'oikos, dont elle était une dimension fondamentale ? L'objet de cet article est de comparer les passages des philosophes de la période classique - Platon, Xénophon et Aristote, mais aussi le Pseudo-Aristote - relevant d'une réflexion sur l'économie domestique, pour observer les déplacements et les recoupements auxquels ils se livrent sur ce thème. Si tous s'interrogent sur le principe moteur de l'union conjugale, sa nature et sa finalité, ils ne les envisagent pas de la même façon. Tous s'accordent néanmoins, avec des différences notables cependant, à voir dans cet aspect de l'économie domestique un processus de fabrique des corps des membres de la cité : au sein de l'économie domestique, le mariage est l'institution médiatrice entre le biologique et le politique.

Mots-clés : Aristote ; corps ; économie ; mariage ; oikos ; Platon ; Xénophon.

Abstract: What did the classical Greek philosophers think about domestic economy, and especially about the conjugal relationship as a fundamental dimension of the oikos? The purpose of this paper is to compare the differences and the overlaps on this topic among the philosophers of the Classical Period - Plato, Xenophon, Aristotle, and also Pseudo-Aristotle - in their texts that deal with domestic economy. If all of them examine the driving principle of the conjugal union, its nature and its purpose, they do not consider them in the same way. However, all agree, though with notable differences, in understanding this aspect of the domestic economy as a process of building the bodies of the members of the city. Marriage, as an important aspect of domestic economy, is revealed as the mediating institution between the biological sphere and the political sphere.

Keywords: Aristotle; body; economics marriage; oikos; Plato; Xenophon. 


\section{Introduction}

L'intérêt pour l'oikos et sa bonne administration est présent dès l'Odyssée et Les Travaux et les Jours. Mais à partir du dernier tiers du Ve siècle, un type d'écrit nouveau et résolument théorique, le logos oikonomikos (DESCAT, 1988, p. 104), en fait son objet propre sous diverses formes : dans des traités séparés, comme l'Économique de Xénophon, l'Économique du Ps. Aristote et l'Économie de Philodème de Gadara $^{1}$; dans des passages précis au sein d'œuvres plus vastes comme les Lois de Platon et les Politiques Aristote ; dans des petits traités qui examinent séparément les grandes questions du genre, comme ceux que Musonius Rufus consacre aux bienfaits de l'agriculture pour le philosophe, ou à la finalité du mariage. ${ }^{2}$ Pour ne pas excéder des limites raisonnables, cet article n'examinera que les textes de la période classique : ceux de Platon, de Xénophon et d'Aristote - ainsi que le traité du Ps. Aristote qui, malgré sa rédaction plus tardive, ${ }^{3}$ porte l'empreinte très nette de ces deux derniers auteurs. L'oikos étant à la fois une communauté matérielle et une communauté humaine - qui ne se limite pas à la famille nucléaire mais qui est, au moins idéalement, centrée sur elle $^{4}$-, ces textes évoquent toujours ces deux aspects, dans le cadre d'un questionnement dont l'architecture demeure assez stable. Concernant les biens, il porte le plus souvent sur les modalités, bonnes et, au contraire, perverses ou fautives, de leur acquisition, de leur conservation et de leur usage, l'accent étant mis sur l'une ou l'autre de ces trois opérations selon les textes envisagés. ${ }^{5}$ Concernant les êtres humains, ce sont les relations du maître avec les esclaves, ainsi que les relations entre l'homme et la femme libres, soit l'époux et l'épouse, qui occupent le cœur de ces réflexions, avec, plus rarement, un examen des rapports entre les parents et les enfants.

\footnotetext{
${ }^{1}$ Ce traité forme le Livre IX de son traité Des Vices et des Vertus qui leur sont opposées, mais peut se lire de façon autonome.

${ }^{2}$ Pour une liste plus complète, voir Natali, 1995. Pour Musonius Rufus, voir King, 2010.

${ }^{3}$ Peut-être entre 325 et 275 av. J.-C. : Wartelle, 1968, p. xii.

${ }^{4}$ Damet, Moreau, 2017, p. 31-32.

${ }^{5}$ Voir Helmer, 2016, p. 196-199 ; et Leshem, 2012.
} 
C'est la relation conjugale entre l'homme libre et la femme libre dans l'oikos que je me propose d'étudier pour montrer qu'elle donnait lieu à de vifs débats chez les philosophes antiques classiques dans le cadre de leur réflexion théorique sur l'économie domestique. Ces mots en italiques permettent de préciser la nature de ma démarche. Tout d'abord, elle ne se concentre pas sur la sexualité conjugale, à laquelle Michel Foucault consacre l'un des chapitres du deuxième volume de l'Histoire de la sexualité ("L'usage des plaisirs »), dans le cadre d'un examen minutieux de certains passages de l'Économique de Xénophon. ${ }^{6}$ Selon lui, la prescription de la fidélité sexuelle évoquée dans ces textes n'obéit pas à une éthique motivée par le lien personnel entre les époux - comme si ces passages préfiguraient la codification chrétienne de la sexualité - mais à un ensemble de normes et de valeurs qui leur sont extérieures et qui régulent leurs conduites respectives dans leur relation. Mon approche est distincte. Elle ne fait pas de la sexualité en général l'objet privilégié de la réflexion grecque sur la relation conjugale - qui soulève aussi, on va le voir, d'autres questions - et elle ne la traite pas comme un objet éthique : elle ne l'évoque que pour ce qui concerne la reproduction et ce qu'elle engage quant au corps des époux et des enfants, afin de comprendre ce que cet aspect du logos oikonomikos nous dit de la pensée antique de l'économie.

Ensuite, est également laissée de côté la question du mariage chez les philosophes ou les sages quand elle n'est pas directement reliée à une préoccupation pour l'administration de l'oikos. La raison de cette mise à l'écart est que les textes consacrés au mariage des philosophes - principalement la République de Platon pour ce qui est de la période classique, si on laisse de côté le cas de Socrate - sont souvent porteurs d'un geste de réforme aboutissant à s'émanciper du cadre de l'oikos, ou à le remodeler, au point qu'il n'a plus de commune mesure avec la structure qu'il présente dans les formes du logos oikonomikos évoquées plus haut. Tel est le cas de la réforme de la famille (plutôt que son abolition) concernant les gardiens philosophes de la République, dans le cadre de la communauté des femmes et des enfants, et du transfert

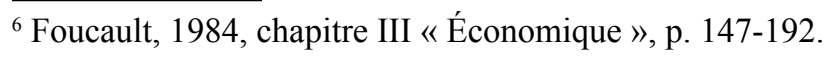


de l'administration des biens matériels à un autre groupe de la cité. ${ }^{7} \mathrm{Tel}$ est aussi le cas, au début de la période hellénistique, du traitement que les cyniques réservent au mariage et à la famille : eux aussi réforment plus qu'ils n'abolissent ces deux institutions - l'union et la paternité de Cratès et de Hipparchia en offrent l'exemple (D. L. VI 88 ; 96-97) - ce qui implique là encore, sous l'effet de la pauvreté et de la mendicité volontaires propres à leur philosophie, une telle dissolution des pratiques usuelles relatives aux biens matériels, que parler d'oikos dans ce cadre s'avère non pas impossible mais très polémique. L'intérêt de ces passages de Platon et des cyniques est indiscutable : ils portent le logos oikonomikos à ses limites et en proposent une autre articulation au nom d'un déplacement du sens des activités ordinaires étudiées dans les traités consacrés à l'oikonomia. Mais précisément pour cette raison, ils auraient davantage leur place dans une étude des alternatives philosophiques au logos oikonomikos que dans cet article.

Enfin, malgré les liens étroits entre les diverses formes du discours à cette époque, ne sont intégrés à cette étude ni les textes des orateurs ni la poésie, notamment la comédie, qui sont pourtant de précieuses sources de connaissance des représentations et de l'imaginaire de l'économie domestique en général, et de la relation matrimoniale en particulier. Outre la difficulté d'intégrer dans un seul texte un corpus aussi vaste, il ne s'agit ici que de rendre compte des problèmes théoriques, des arguments et des concepts qui animaient le débat philosophique sur cette question.

Concernant la méthode, plusieurs remarques sont nécessaires. D'une part, la forte teneur idéologique de ces textes consacrés à la relation conjugale est indéniable et peut à juste titre paraître scandaleuse aux lecteurs contemporains. Les arguments qu'ils mobilisent sont souvent destinés à justifier, par exemple en la naturalisant, la position inférieure de la femme par rapport à l'homme. C'est à l'évidence le cas de Xénophon, d'Aristote et du Ps. Aristote; et si Platon est assurément le plus critique envers ce cadre idéologique dont il met à nu les rouages et la violence, il n'y échappe pas totalement non plus. Il est important d'en avoir conscience, mais je n'y reviendrai pas dans la suite, notamment au

\footnotetext{
${ }^{7}$ Sur le remodelage de l'oikos dans la République, voir Helmer, 2011.
} 
moment d'évoquer la distribution des tâches, où cet aspect est le plus marqué.

D'autre part, plutôt qu'une étude par auteur, la démarche adoptée ici consiste à comparer, en repérant les recoupements et les déplacements dont ils font l'objet, les problèmes, arguments et concepts que ces auteurs mobilisent lorsqu'ils traitent de la relation conjugale dans le cadre du logos oikonomikos. Si tous lui reconnaissent un rôle clé dans l'édification d'un monde humain défini par ses institutions principales - l'éducation, la famille, la cité et, en un sens, la nature - ils n'en font pas simplement la condition mécanique de possibilité de toutes ces institutions : elle est aussi à leurs yeux le point de convergence ou de cristallisation qui leur donne leur forme singulière. Quelles sont donc les grandes questions que posent ces auteurs à propos et à partir du mariage, et qui engagent leur idée de l'économie ? On peut en dénombrer trois : quels sont les principes de l'union conjugale, c'est-à-dire les causes motrices expliquant la formation des couples de sexes opposés? Quelle est la nature de l'union ainsi formée ? Enfin, quelle en est la finalité ?

\section{Principes moteurs de l'union : de la nature à la cité}

Comment penser le mariage ? Est-ce une institution dont les fondements sont à chercher du côté de la nature, ou est-ce une création artificielle appelée par la nécessaire inscription de l'homme dans l'espace de la cité ? À y regarder de près, cette alternative est trop moderne et demande à être précisée. Car la sphère politique et la sphère naturelle ne sont pas nécessairement antagonistes chez les philosophes qui nous occupent : en réalité, elles sont le plus souvent en accord l'une avec l'autre. Ainsi, Platon évoque une politique « vraiment conforme à la

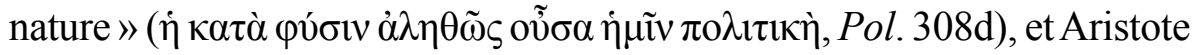
fait de la cité la communauté naturelle achevée (Polit. 1252b30-1253a2). La question est donc plutôt de savoir ce que désigne cette nature pour les uns et les autres, de comprendre comment le mariage articule ces deux sphères, et comment lui-même reçoit de cette articulation sa coloration particulière chez ces différents auteurs. L'examen du principe moteur de l'union des sexes opposés dans le mariage permet de saisir les diverses 
modalités de cette articulation chez les auteurs qui nous occupent. On peut distinguer trois cas de figures.

\subsection{L'enracinement biologique}

Le premier, propre à Aristote et en partie au Ps. Aristote, est celui de l'enracinement biologique, dans le cadre de la finalité naturelle, du rapprochement du mâle et de la femelle humains. C'est en considérant d'abord l'être humain comme une espèce animale confrontée à la nécessité de se perpétuer qu'Aristote explique le rapprochement des sexes à l'origine de l'oikos :

Ainsi, il est nécessaire tout d'abord que s'unissent

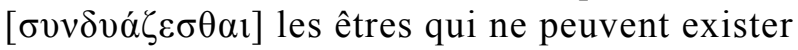
l'un sans l'autre, par exemple la femelle et le mâle en vue de la procréation $[\theta \tilde{\eta} \lambda v \mu \varepsilon \grave{\varepsilon} v \kappa \alpha i ̀ ~ \alpha ̊ \rho \rho \varepsilon v \tau \tilde{\eta} \varsigma$

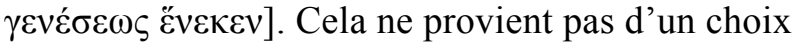

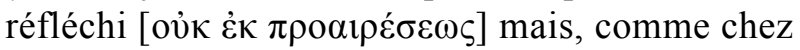
les autres animaux et les plantes, de la tendance

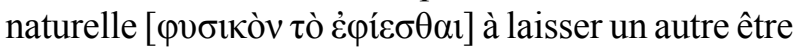
tel que soi. (ARISTOTE, Politiques 1252a26-31; trad. P. Pellegrin modifiée).

Aristote n'ignore pas que les individus humains peuvent choisir de ne pas marier et de ne pas avoir d'enfants : mais pour rendre raison de la perpétuation observable de l'espèce et des institutions que sont les maisons et les cités, il faut supposer qu'une tendance souterraine est à l'œuvre, dont les individus sont moins les agents que les instruments. Cet ancrage naturel et nécessaire de l'union des sexes à l'origine de l'oikos est aussi évoqué par le Ps. Aristote : selon lui, « la communauté

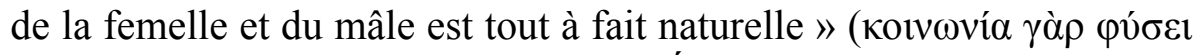

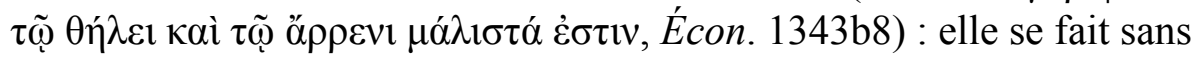
l'intervention de la raison pour ce qui concerne la procréation, et elle donne lieu en outre à des conduites d'assistance et d'aide mutuelle chez les animaux apprivoisés et plus intelligents (1343b13-18). À l'évidence, le Ps. Aristote complète ici l'exposé d'Aristote en combinant les données de sa politique et de sa biologie : il associe l'ancrage naturel de l'union 
en vue de la procréation, soit le souci du vivre, à l'existence de relations « conjugales » plus complexes qui assoient les prémisses du bien-vivre - sous la forme de l'assistance et de l'aide - ouvrant ainsi à la finalité éthique de la politique. On verra toutefois que l'ancrage biologique de la relation conjugale chez le Ps. Aristote est moins catégorique que chez Aristote, dans la mesure où pour le premier, il dépend en dernier ressort, comme chez Xénophon (VII 18), de l'intervention d'un dieu qui fait de la nature l'instrument de ses projets.

Cette approche biologique explique que la législation des mariages proposée par Aristote - législation qui doit assurer, par des unions réussies, la procréation d'enfants doués de la « bonne disposition

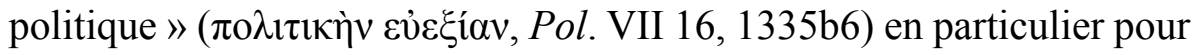

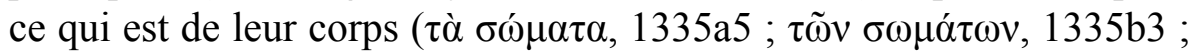

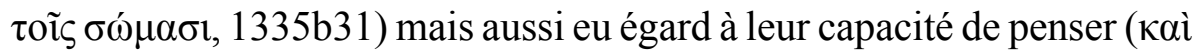

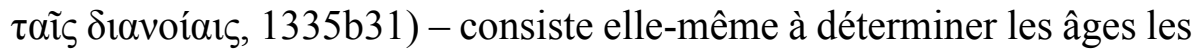
plus appropriés au mariage pour l'homme et la femme, en tenant compte

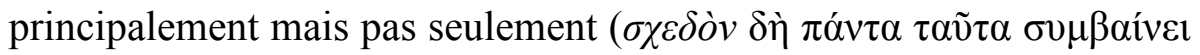

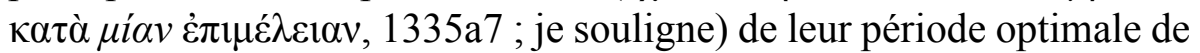
fécondité.

\subsection{Un bon parti}

Pourtant, la nature suffit-elle à rendre raison des unions ? Un deuxième principe, de nature sociale et économique, et qui devait correspondre à une réalité de l'époque, doit être examiné pour en rendre compte : trouver un bon parti. C'est ce que Xénophon évoque dans un entretien d'Ischomaque avec sa femme :

Dis-moi, ma femme, as-tu compris maintenant à quelle fin je t'ai épousée et à quelle fin tes parents t'ont donnée à moi ? Nous n'étions pas embarrassés, ni toi ni moi, de trouver quelqu'un avec qui dormir : tu t'en rends bien compte, je le sais, tout comme moi.

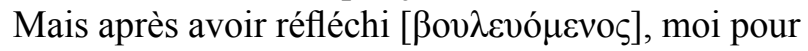
mon propre compte, et tes parents pour le tien, au meilleur associé que nous pourrions nous adjoindre 


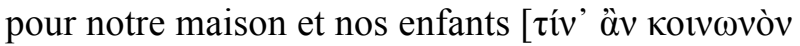

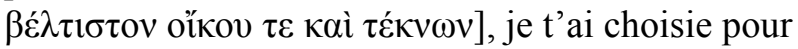
ma part, et tes parents, il me semble, m'ont choisi moi, parmi les partis possibles [ $\dot{\varepsilon} \kappa \tau \tilde{\omega} v \delta v v \alpha \tau \tilde{\omega} v$

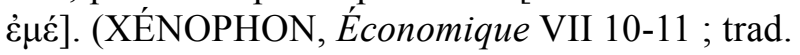
P. Chantraine).

Le principe de l'union, exprimé au niveau de la conscience qu'en ont ses agents, relève ici d'une préoccupation sociale et économique au sens matériel, qui conduit les individus à délibérer et à décider

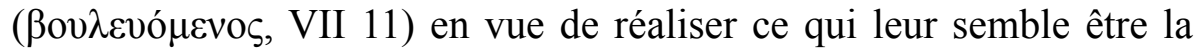
meilleure « communauté » domestique qui soit, c'est-à-dire, comme

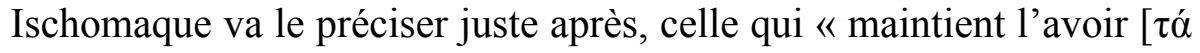

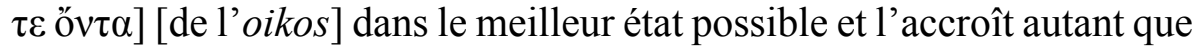
possible par des moyens honorables et légitimes » (VII 15). Consolider et renforcer la valeur de l'oikos était sans doute une motivation explicite à l'époque classique, ce à quoi une autre traduction possible de l'expression

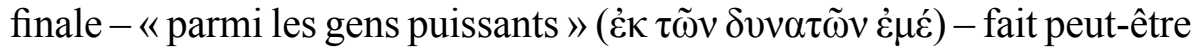
allusion. C'est ce qui permet de comprendre ce qui, sans cela, pourrait passer pour la mention d'un sentiment personnel de l'épouse envers l'époux envisagé comme personne singulière : si Ischomaque était fardé, son épouse serait, dit-elle, « incapable de le chérir de toute [s]on âme »

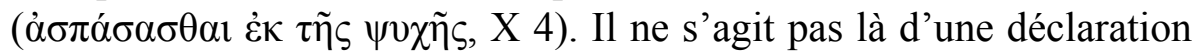
d'amour de la femme à son mari considéré en tant qu'individu particulier, mais de l'attachement à un partenaire dans la communauté de l'oikos, comme le contexte de cette citation, qui concerne « la communauté des corps », permet de le voir : de même que les espèces du cheval et du mouton ne trouvent rien de plus agréable que le corps du cheval et du mouton, de même « les hommes ne trouvent rien de plus agréable que le

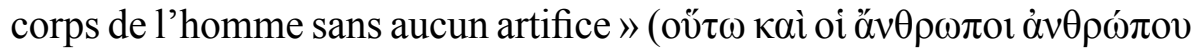

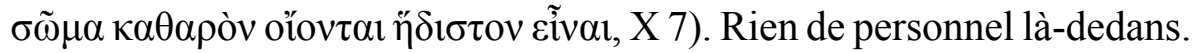
On y reviendra à propos de la finalité de l'union conjugale.

Cette intention consciente qui préside aux unions représente, dans le discours d'Ischomaque, un point de convergence de la nature, de la religion et de la coutume : les trois institutions fondamentales par lesquelles l'homme trouve sa place dans l'univers vont toutes dans le 
même sens. En effet, les dieux, explique-t-il, ont différencié naturellement le corps de l'homme et de la femme en vue des fonctions économiques qui

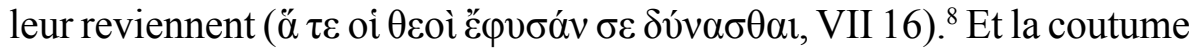
(ó vó $\mu$ os, VII 16 et 30) confirme une telle distribution des aptitudes et des fonctions. Xénophon témoigne ainsi d'un système idéologique où tout converge en vue de ce qui semble avoir été la pratique, réelle ou idéale, d'une certaine économie, qui laisse son empreinte sur l'idée et la pratique du mariage.

\subsection{Une stratégie sociale, économique et politique mise à nu}

Platon, enfin, propose une troisième sorte d'approche, distincte de celle d'Aristote et de celle de Xénophon, et nettement critique à leur égard, notamment envers le second. Loin de considérer, comme Aristote, que les fondements naturels de la relation conjugale, à savoir la différence et la complémentarité des sexes en vue de la procréation, sont déterminants pour rendre raison de l'unité domestique dans sa dimension conjugale, Platon les subordonne à l'édification d'un ordre politique qui prime en tant qu'institution fondamentale par laquelle l'homme se réalise ou, au contraire, se détruit. Le moteur de l'union des sexes dans la relation conjugale telle qu'elle a lieu le plus souvent n'est pas, à ses yeux, la nécessité biologique générale que l'espèce a de procréer : c'est un ensemble de stratégies sociales préjudiciables à la communauté politique et dont les agents ne mesurent pas toutes les conséquences. Elles forment en quelque sorte le moteur secret ou la toile de fond du discours tenu par Ischomaque chez Xénophon, dont Platon dévoile toutes les implications pour la cité et ses membres. Régler les unions consistera donc pour Platon à régler ces stratégies au nom du bien politique qu'est l'unité harmonieuse de la cité juste. ${ }^{9}$

\footnotetext{
${ }^{8} \mathrm{Cf}$. VII 18 : « les dieux ont procédé à un examen approfondi avant d'assortir ce couple qu'on appelle mâle et femelle pour le plus grand avantage de leur communauté »; et VII 22 pour les travaux, respectivement, de l'extérieur, de l'intérieur.

${ }^{9} \mathrm{C}$ 'est pourquoi les passages dans lesquels Platon évoque ces questions ne sont pas des traités économiques comparables à ceux de Xénophon ou du Ps. Aristote : il déplace le logos oikonomikos sur le terrain politique en l'intégrant dans la République, les Lois et le Politique.
} 
Platon part en effet du constat que, dans les cités empiriques et imparfaites dans lesquelles nous vivons, les unions sont mal réalisées, au sens où leur principe œuvre contre l'unité de la polis, c'est-à-dire contre la réalisation d'une communauté d'intérêts et d'affects. Deux motifs principaux guident ces unions. Le premier, comme l'a exprimé Ischomaque et comme cela se produisait à Athènes, ${ }^{10}$ est le désir de former des familles puissantes par leur richesse ou leur pouvoir. Un passage du Politique et un passage des Lois le signalent clairement :

L'Étranger - [...] La plupart du temps, en effet, ces liens [du mariage] sont noués d'une façon qui ne convient pas à la procréation des enfants.

Socrate le Jeune - Quoi donc?

L'Étranger - Quand, en ces occasions, ce sont des

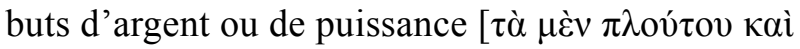

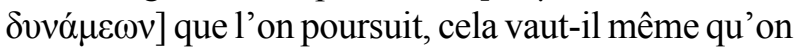
prenne la peine d'en parler pour les blâmer?

Socrate le Jeune - Nullement. (PLATON, Politique $310 \mathrm{~b}$; trad. L. Brisson, J.-F. Pradeau).

L'Athénien - Il [te] faudra contracter le mariage qui méritera l'approbation des gens réfléchis, qui te recommanderaient de ne pas fuir l'alliance avec les pauvres, et de ne pas trop chercher à te marier chez les riches. Il faut plutôt, toutes choses égales par ailleurs, toujours préférer le parti le moins opulent et s'engager dans cette union. La cité y trouvera son avantage, de même que les foyers qui résultent de ces unions [ $\tau \alpha$ ĩ

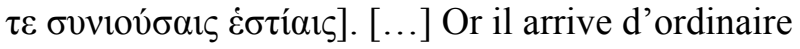
que chaque nature se porte vers ce qui lui ressemble le plus, d'où naît pour la cité dans son ensemble un déséquilibre tant des fortunes que des façons de vivre

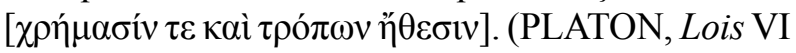
773a-c ; trad. L. Brisson, J.-F. Pradeau).

\footnotetext{
${ }^{10}$ Voir Cox, 1998, en particulier p. 38 : « Once two families were united in marriage, kinsmen and kinswomen acted so as to reinforce those ties and consolidate property and wealth. »
} 
Tandis que le Politique, on le voit, se contente de signaler ce problème sans le traiter, les Lois proposent un certain nombre de dispositions pour éviter que le jeu des intérêts d'argent ne perturbe les unions, au nom de l'équilibre et de l'unité de la cité. Outre des dispositions générales - la richesse est placée d'emblée au dernier rang des biens humains, loin derrière les biens divins (Lois I 631b-d), et la richesse (comme la pauvreté) fait l'objet d'une limitation pour tous les citoyens (Lois V 744d-745b) - une mesure plus spécifique, l'interdiction de la dot, vise directement le mariage :

L'Athénien - [...] il faut interdire les dots, et expliquer aux pauvres que prendre femme sans dot ne condamne pas à mal vivre par manque de richesses. Car dans la cité qui est la nôtre, personne ne manque du nécessaire; et les richesses porteront moins les épouses à faire preuve d'insolence et les maris de servilité basse et déshonorante. (PLATON, Lois VI 774c-d ; trad. L. Brisson, J.-F. Pradeau).

Interdire la dot, c'est donc soustraire le mariage à des effets moraux qui peuvent le déstabiliser, et avec lui toute la cité. C'est aussi rappeler au citoyen que le mariage est avant tout une institution politique, dont il n'est que l'instrument ou l'exécutant au nom d'un bien collectif dont il recevra les bénéfices s'il suit les prescriptions du législateur.

Le second motif qui, selon Platon, guide d'ordinaire les unions, est à la fois psychologique et politique. Il concerne la propension des deux caractères principaux présents dans la cité - les courageux et les modérés - à vouloir s'unir avec ceux qui leur ressemblent plutôt que de s'unir entre eux :

L'Étranger - Ceux qui sont modérés [oi $\mu \varepsilon ́ v \pi$ rov

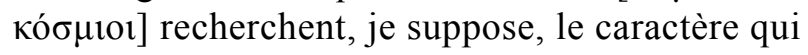
est le leur, et prennent autant que faire se peut, leurs femmes dans ce milieu, et quand inversement ce sont eux qui marient leurs filles, ils les conduisent à des maris de ce même milieu. Et c'est du reste ainsi que font aussi ceux qui appartiennent au groupe 


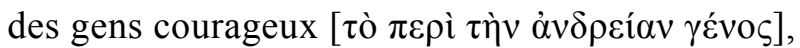
voulant retrouver leur propre naturel, alors que les deux groupes devraient faire le contraire. (PLATON, Politique 310c-d ; trad. L. Brisson, J.-F. Pradeau modifiée)

Tendances à la fois naturelles et modelées par l'éducation, ces deux caractères soutiennent deux vertus, le courage et la modération, qui, malgré leur commune nature de vertu, se trouvent néanmoins en conflit l'une avec l'autre (Pol. 306a-308b). Cette contrariété se révèle surtout à propos « des choses importantes »- les affaires politiques - où elle devient « une maladie, la plus détestable dont puissent souffrir les cités » (Pol. 307e) : cultivée sans mélange, chacune d'elle mène la cité à la ruine et à la servitude, soit en la rendant incapable de se défendre (la modération), soit en lui créant trop d'ennemis (le courage) (307e-308a). Sous l'effet de la tendance spontanée à s'unir avec le ressemblant, ces deux vertus tendent ainsi à devenir des vices - respectivement la folie et la nonchalance (310d-e) - néfastes pour la cité. Seule une opinion partagée par les deux groupes sur « le bien et le mal » (310e) - c'est le « lien divin » que doit façonner le politique - peut assurer leur mélange par le mariage qu'est le « lien humain » $(310 \mathrm{e}-311 \mathrm{a}),{ }^{11}$ et garantir ainsi leur coopération à tous les niveaux de la cité, surtout pour les magistratures (311a), qui sont si importantes pour son bon gouvernement. Que le dialogue portant sur la définition du politique et de la politique viennent se clore sur l'analyse du mariage dit assez bien que l'union conjugale, et avec elle une dimension fondamentale de l'économie domestique, est pour Platon un sujet avant tout politique. De la façon de se marier dépend le destin de la cité.

Les différences d'accents sont donc notables chez ces auteurs dans l'analyse des principes du mariage, et Platon est à l'évidence très critique envers le dispositif idéologique naturaliste d'Aristote, et celui, social, de Xénophon. Qu'en est-il dès lors de la nature de l'union conjugale ? Ces auteurs la conçoivent-ils tous de la même façon?

${ }^{11}$ La nécessité du même mélange est formulée dans les Lois VI 773a-b. 


\section{La nature du lien conjugal dans l'oikos : versions de la communauté}

C'est sous le signe de la communauté qu'ils présentent l'union conjugale. Pour chacun d'entre eux, l'homme et la femme dans l'oikos forment une koinônia, ${ }^{12}$ et chez Xénophon, le partenaire est plusieurs fois appelé un koinônos (III 15 ; VII 11 et 13). Mais quel est l'objet de cette communauté ? Si la nature des fonctions dévolue à l'homme et à la femme est assez semblable dans la plupart les cas, deux idées de la communauté se font face - Platon restant un cas à part sur ces deux points.

\subsection{L'oikos, lieu et principe de communauté}

De nombreuses occurrences du vocabulaire de la communauté (koinônia, koinônos, koinos) figurent dans le texte de Xénophon pour qualifier l'union de l'homme et de la femme. Plus précisément, c'est l'oikos qui, pour Ischomaque, est à la fois le lieu et le principe de cette communauté : " Pour le moment », explique-t-il à sa femme, " c'est cette maison seule qui nous est commune » (võv $\delta \dot{\varepsilon} \delta$ ò̃ oĩ

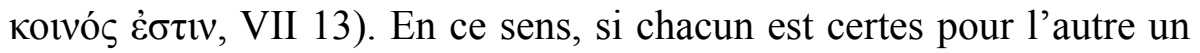
« partenaire » (koinônos), il l'est avant tout, littéralement, de l'oikos

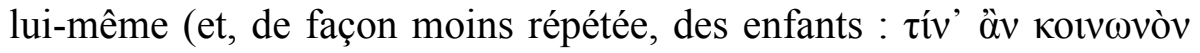

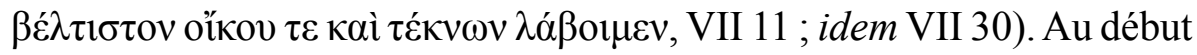
de l'ouvrage, Socrate laissait déjà entrevoir à Critobule qu'une femme

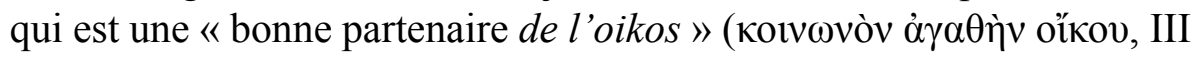
15 ; je souligne) équivaut à l'homme sur ce plan là. L'union des époux n'a donc de sens que par la médiation de cette institution : ils ne sont pas communs l'un envers l'autre, mais envers l'oikos qui les rend communs.

C'est ce que signalent les quelques termes formés sur le préfixe sun-, qui évoquent une action accomplie ensemble : Socrate propose à Critobule de lui expliquer comment la femme peut être employée comme

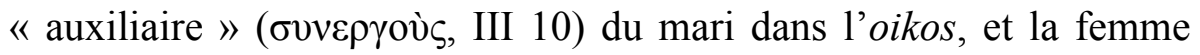

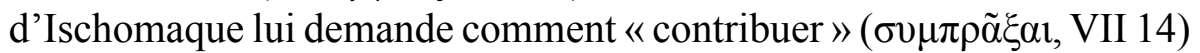
aux activités domestiques, ce à quoi Ischomaque répond en l'invitant

${ }_{12}$ Voir Lois VI 773a et 773d ; Politiques $1252 \mathrm{~b} 10$; Économique de Xénophon VII 18 ; Économique du Ps. Aristote III 1, 1343 b27. 


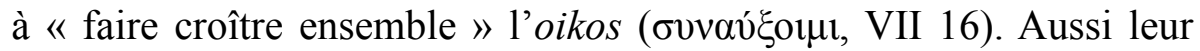
demande conjointe aux dieux de ce qui peut être « le mieux pour tous

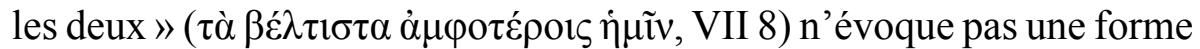
de réciprocité entre Ischomaque et son épouse mais, comme le signale le duel, leur commune participation à l'oikos; ou plus exactement, c'est l'oikos qui rend commune cette participation et fait qu'elle les concerne tous les deux.

Que l'oikos soit le principe unique de la communauté entre les époux n'implique pas qu'ils y jouent le même rôle. C'est au contraire leur complémentarité fonctionnelle qui prévaut : elle est rendue possible par la différence naturelle de leur corps (VII 18-22) et de leurs aptitudes morales (VII 24-25 : tendresse et bravoure), à laquelle les dieux ont

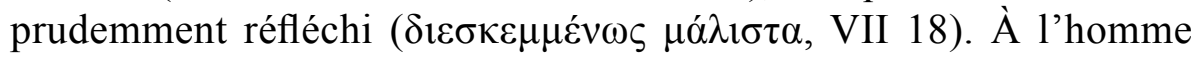
l'extérieur, le soin de faire entrer les provisions et de défendre l'oikos ; à la femme l'intérieur, le soin de garder ces provisions, de les distribuer comme il convient (VII 39-40), et de prodiguer ses soins aux nouveaunés (VII 24). Cette complémentarité permet de comprendre que la communauté des corps évoquée en X 4-5 à propos de la question de

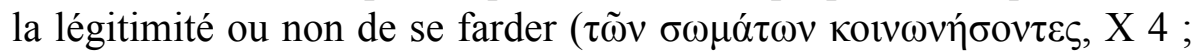

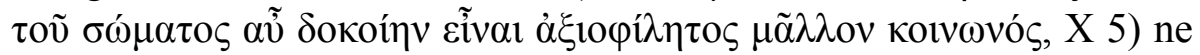
désigne pas l'union sexuelle mais la complémentarité physique et, par là, fonctionnelle, de l'homme et de la femme, dont l'union sexuelle n'est qu'une modalité possible. Le corps économique est le corps spécifique - de l'espèce -, non le corps individuel. L'analogie d'Ischomaque, déjà citée plus haut, le dit bien : de même que les dieux ont fait du corps des chevaux, des bestiaux et des moutons la chose la plus agréable du monde pour les chevaux, les bestiaux et les moutons, c'est-à-dire pour les membres de leur espèce, de même « les hommes ne trouvent rien de plus agréable que le corps de l'homme sans aucun artifice » (oṽ $\tau \omega$

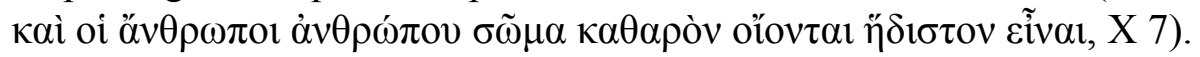
Et le conseil d'Ischomaque à sa femme pour rester belle le confirme : il ne consiste pas à l'aider à faire ressortir sa beauté individuelle, mais à remplir au mieux son rôle dans l'oikos (X 9-13). Toujours active, elle aura ainsi non pas une apparence de beauté mais une beauté véritable : non pas celle de sa personne - car elle n'est personne pour ainsi dire 
(son nom n'est pas même mentionné) - mais celle qui fait transparaitre et reconnaître en elle un ordre social à maintenir.

\subsection{La communauté des époux}

S'ils font également de la relation conjugale une communauté, Aristote et, jusqu'à un certain point le Ps. Aristote, en précisent toutefois la notion par rapport à Xénophon : ils adoptent la même distribution des fonctions, avec plus ou moins d'emphase, mais proposent une autre idée de la communauté.

Dans une reprise évidente mais abrégée d'éléments de l'Économique de Xénophon, le Ps. Aristote conçoit lui aussi la communauté domestique formée par l'homme et la femme (1343b27) comme une coaction ( $\sigma v v \varepsilon \rho \gamma \grave{\alpha}, 1343 b 20$ ) en vue du bien-vivre, enracinée toutefois, comme pour Aristote, dans la communauté biologique du mâle et de la femelle. Là encore, comme l'indique une expression inspirée de Xénophon, ${ }^{13}$ la divinité a organisé la nature de manière à différencier les corps et les fonctions qui leur reviennent, dans le cadre d'une complémentarité similaire à celle exposée par Ischomaque : l'homme étant fait plus fort, la femme plus faible (1343b30), celle-ci est plus apte à garder ou monter la garde sous l'effet de la crainte qui l'anime, celui-là plus apte à repousser l'attaque par son courage ; il est plus apte à amener ce qui vient du dehors, elle est plus apte à sauvegarder ce qui est à l'intérieur ; son travail à elle sera plus sédentaire, son travail à lui, qui est moins doué pour la tranquillité, lui fera trouver la santé dans le mouvement ; quant aux enfants, si la genèse en est commune, elle les nourrit, il les éduque (1343b23-1344a6). Aristote, pour sa part, s'étend très peu sur le détail de cette répartition des fonctions : il se contente d'indiquer que " son rôle à lui est d'acquérir, son rôle à elle de conserver »

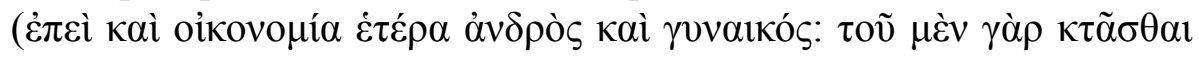

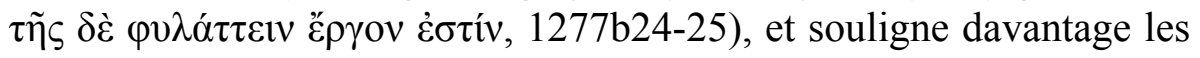

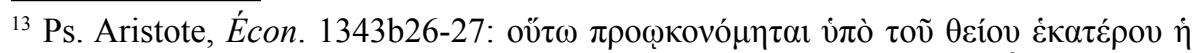

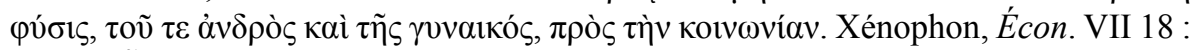

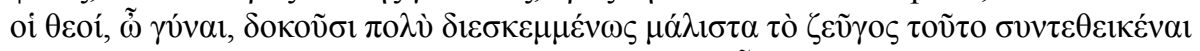

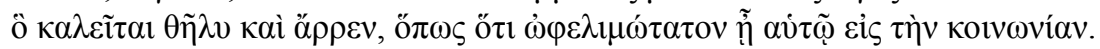


différences de pouvoir entre les époux : l'homme et la femme dans l'oikos diffèrent en hiérarchie (1259a37-1259b2), parce que leurs âmes possèdent selon lui les mêmes parties mais différemment. Elles ont donc part aux mêmes vertus mais de façon différente (1260a9-21).

Cependant, le point véritablement important qui distingue Aristote et le Ps. Aristote d'un côté, de Xénophon de l'autre, concerne la nature de la communauté : elle a lieu désormais directement entre l'époux et l'épouse, et n'est plus médiatisée par l'oikos. C'est ce qui explique - ou s'explique par-la prise en compte de leur relation éthique, fondée à la fois sur la morale commune (ó kotvòs vó sur des considérations psychologiques. Examinons ces deux points. Concernant la morale commune, le Ps. Aristote évoque l'importance de respecter la justice, et plus exactement de ne pas commettre l'injustice envers l'épouse, qui est particulièrement vulnérable - ce que suggère la référence aux Pythagoriciens selon qui la femme est « une suppliante, arrachée à son foyer » (1344a9-10). L'injustice à laquelle songe l'auteur désigne manifestement les « relations hors du foyer » évoquées juste

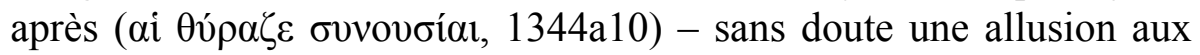
relations sexuelles extraconjugales. Dans ce contexte, quel sens donner

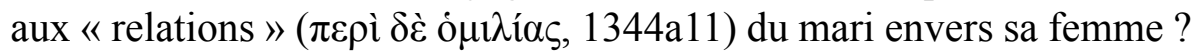
Faut-il y entendre un sens sexuel, qui répondrait à la remarque précédente sur les « relations hors du foyer $» ?{ }^{14}$ Dans ce cas, le Ps. Aristote voudrait dire que le mari doit agir sur ce plan envers sa femme de manière à ce qu'elle ne soit ni en demande quand il est présent, ni être incapable d'être en repos quand il est absent. Ou bien faut-il prendre la formule en un sens « fonctionnel », plus directement lié aux tâches domestiques : la femme doit savoir être autonome dans l'exécution des tâches, aussi bien quand son mari est là que quand il ne l'est pas ? Dans un cas comme dans l'autre, on notera que le Ps. Aristote n'emploie pas le terme positif de « justice » à propos de cette relation, comme si l'important était de

\footnotetext{
${ }^{14}$ Ce que propose J. Tricot : « En ce qui concerne les rapports intimes des époux, une femme ne doit ni s'en passer complètement ni être incapable de demeurer en repos en l'absence du mari, mais le mari doit accoutumer sa femme à être également satisfaite présent ou absent. »
} 
ne pas entamer ou grever la relation, plutôt que de se mettre en quête de la nature de la justice au sens plein du terme dans cette même relation. Signe de cette distance par rapport à une définition positive de la justice, la mention succincte d'une forme de philia entre les époux ( $\varphi \imath \lambda \imath \kappa o ́ v$, 1344a15), que doit favoriser la similitude de leurs caractères (1344a16).

Aristote évoque plus directement ces éléments éthiques dans un passage de l'Éthique à Nicomaque. La philia qui unit les époux peut se déployer selon un vaste registre. Fondée en nature, du fait que l'être

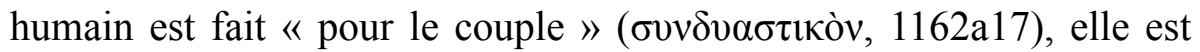
susceptible des trois modalités de toute amitié : l'utilité, par laquelle « chacun supplée aux besoins de l'autre en mettant en commun les

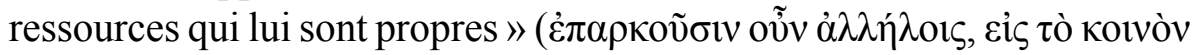
$\tau \imath \theta \varepsilon ́ v \tau \varepsilon \zeta \tau \grave{\alpha}$ ídı, 1162a19) - expression qui signale que la communauté tient à la relation entre les époux plutôt qu'elle n'est médiatisée par l'oikos - ; le plaisir et, enfin, la vertu, du moins si les époux sont « convenables »

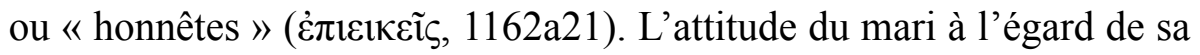
femme doit consister à se demander « comment faire ce qui est juste »

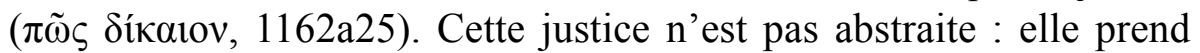
la forme d'une relation de pouvoir particulière, propre à la relation maritale, qu'Aristote définit comme « politique », au sens où le rapport est d'égalité entre gouvernant et gouverné (Politiques 1255b20), ici entre l'homme libre et l'épouse libre. Ce type de relation implique en général l'alternance dans l'exercice du commandement (1259b4-6). Pourtant, ce qui vaut pour la cité ne vaut pas pour l'oikos: Aristote prête à l'homme une faculté naturelle de commander qu'il n'accorde pas, ou moins, à la femme (1259b2), ce qui va contre l'alternance. Pourquoi dès lors appeler « politique » leur relation si elle ne laisse pas de place à l'alternance dans l'exercice du pouvoir dans l'oikos ? Sans nier-mais jusqu'à quel point? - l'égalité du mari et de la femme, Aristote estime que l'exercice du pouvoir est indiscernable de signes de pouvoir (1259b7-8) : on peut donc supposer (le texte n'en dit rien) que l'homme exhibe naturellement ces signes, plus que la femme en tout cas, peut-être en raison de sa constitution physique d'ordinaire plus forte. Les Magna Moralia proposent une autre explication, procédant par comparaison avec les autres relations internes à 
l'oikos que sont la relation paternelle et la relation despotique : « Le juste dans l'association du mari et de la femme se rapproche beaucoup de la justice politique. La femme sans doute est inférieure à l'homme; mais elle lui est plus intime que l'enfant ou l'esclave; et elle est plus près qu'eux d'être l'égale de son mari. Aussi, leur vie commune se rapproche-t-elle de l'association politique ; et par suite, la justice de la femme à l'époux est en quelque sorte plus politique qu'aucune de celles que nous venons d'indiquer » (1194b22-29). ${ }^{15}$ Dans tous les cas, le Ps. Aristote, et plus encore Aristote, déplacent le point central de la communauté domestique de l'oikos: elle n'est plus une entité surpomblante mais la relation entre les époux eux-mêmes.

\subsection{Platon : l'oikos politique et la similitude des fonctions}

Où se situe Platon dans cette configuration? Il n'en suit pas le modèle, en n'évoquant ni la question de la distribution des fonctions entre les époux ni celle de la justice entre eux. Pourquoi ? C'est de nouveau l'inscription polémique et critique dans la cité de ce que les autres auteurs attribuent à l'oikos qui l'explique, soit l'ancrage politique de l'économique. Concernant le lieu d'application de la distribution des fonctions, Platon non seulement le déplace de l'oikos vers la polis, au moment d'exposer la genèse de la cité au livre II de la République ( $369 \mathrm{~b} s q$.) mais il en change aussi le critère : il n'est plus sexuel, comme chez les auteurs précédents, mais relatif au talent ou à la compétence (370a-b) ; et rien, dans les Lois, n'invalide cette idée. Dans ces deux dialogues, Platon met au contraire en avant la similitude fonctionnelle de l'homme et de la femme au nom de l'unité et de la force de la cité, que ce soit entre gardiens et gardiennes dans la République (V 455d-e) ou, pour ce qui nous occupe ici, entre époux dans l'espace domestique : dans les Lois, l'Athénien exige « que pour

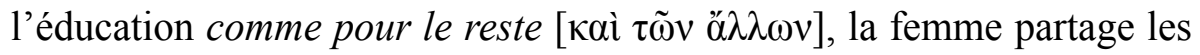
activités des hommes » (VII 805c-d; je souligne), ce qu'il précise ensuite en excluant d'adopter la pratique athénienne consistant «à ramasser toutes

${ }^{15}$ Je traduis en français la traduction anglaise de W. D. Ross, The Works of Aristotle. Magna Moralia, Ethica Eudemia. De Virtutibus et Vitiis, Oxford, Clarendon Press, 1915. 
nos richesses en une seule demeure, et [à les] confier à l'intendance des femmes en y ajoutant le tissage et le travail de la laine dans son ensemble » (VII 805e). Concernant la justice entre les époux, dans les Lois, Platon ne l'évoque pas car il subordonne la communauté domestique à la communauté d'affects et de valeurs qu'est la cité elle-même : les membres du foyer lui appartiennent avant de s'appartenir à eux-mêmes (VII 804d ; XI 923b). Platon évoque toutefois deux mesures destinées à favoriser le lien conjugal. La première porte sur la relation entre les futurs époux au moment de se choisir : ils sont invités à développer

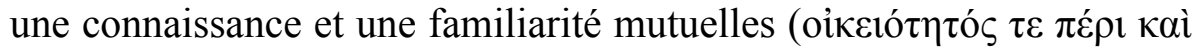
$\gamma v \omega \rho i ́ \sigma \varepsilon \omega \varsigma$ à $\lambda \lambda \eta \dot{\lambda} \omega \mathrm{v}$, Lois, VI 771d), dont les bases sont jetées lors de danses communes où chacun a le loisir d'observer le corps de l'autre. La seconde mesure consiste à mettre en place des repas en commun : destinés à arracher à la réclusion domestique les femmes « habituées à vivre retirées et dans l'ombre » (VI 781c), ils doivent en quelque sorte les politiser, ${ }^{16}$ et par là les inscrire dans la communauté civique dont leurs maris sont aussi membres.

Reste à se demander quelle est la finalité de l'union conjugale. Là encore, des différences d'accents chez les auteurs qui nous occupent sont révélatrices du sens qu'ils donnent à l'économie domestique.

\section{L'économie ou la fabrique des corps}

Quelle est la finalité de l'union conjugale dans l'oikos? Elle est de produire des corps : non seulement d'en créer qui soient d'un type déterminé - c'est le corps des nouveau-nés, sur lequel Aristote et Platon font porter leur réflexion dans le cadre de considérations eugénistes complexes - mais aussi de penser, et de rendre opératoires dans l'espace domestique, les différences et les ressemblances entre les corps des époux, ce que fait Xénophon, qui ne donne à la procréation qu'un rôle secondaire dans ses analyses. Dans tous les cas, l'oikos est une institution dont la juste administration doit produire un certain corps : le corps domestique, sur lequel doit se fonder le corps civique ou politique, parce que c'est

${ }^{16}$ Voir Helmer, 2010, p. 247-249. 
de lui que dépendent en grande partie la permanence de l'oikos et celle de la cité.

\subsection{Acquérir des corps d'homme et de femme}

Xénophon fait certes de la procréation un objet ou une fin de la relation que la divinité a prévue entre l'homme et la femme en organisant la différence de leurs corps et de leurs aptitudes. Ayant reçu la charge de nourrir les enfants, la femme bénéficie également, dans le partage des caractères, de plus de tendresse que l'homme à l'égard des nouveau-nés (VII 24). Mais deux points signalent que c'est le corps des époux qui est au centre de la pratique (et du discours) économique, plutôt que celui du nouveau-né.

D'une part, Ischomaque ne dit pas directement ou simplement que la divinité a conçu le corps de la femme pour donner naissance à des enfants et les nourrir : c'est bien plutôt pour exercer des tâches spécifiques dans l'oikos que la divinité a formé son corps et ses aptitudes. Selon Ischomaque, le corps de la femme, plus vulnérable, est fait pour les tâches

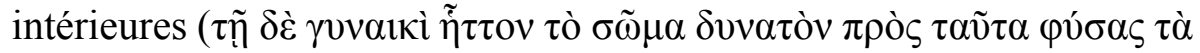

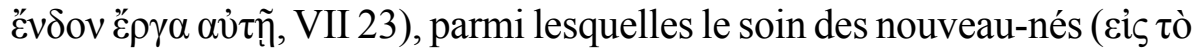

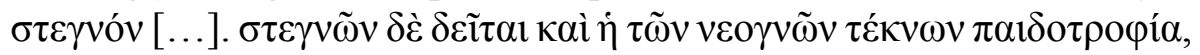
VII 21) n'est qu'un des trois exemples (avec la préparation de la farine et la confection des vêtements). En d'autres termes, Ischomaque ne fait pas de la procréation le point central de ses recommandations à sa femme, ce que confirment à la fois le faible nombre de ses commentaires à ce sujet, ${ }^{17}$ et ce propos : « si la divinité nous en donne un jour » ( $\tau \varepsilon \dot{\kappa} \kappa v \alpha \mu \grave{\varepsilon} v$

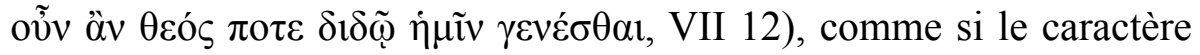
aléatoire ou contingent de la chose - peut-être une référence à la mortalité infantile probablement élevée de l'époque ? - ne permettait pas d'en faire un facteur stable sur lequel compter pour assurer la perpétuation et la prospérité de l'oikos (ce qui, par contraste, expliquerait en partie le détail des considérations eugénistes de Platon et plus encore d'Aristote). Pour Xénophon ou ses personnages, avoir des enfants peut certes représenter

${ }^{17}$ Socrate ne l'évoque pas du tout dans son entretien avec Critobule. 
une aide pour les vieux jours d'Ischomaque et de son épouse (VII 19 ; idem chez le Ps. Aristote 1343b21-23), ainsi que pour souder leur

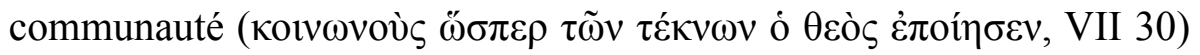
mais ce n'est pas le point essentiel de cette union.

D'autre part, l'objectif explicite de l'union conjugale, « conserver l'avoir et l'augmenter » (VII 15), passe davantage par la distribution et la réalisation efficaces des compétences des époux que par le soin à accorder aux enfants. La justification de cette distribution tient, on l'a vu, à la différence, prévue par la divinité, des corps et de leurs aptitudes (secondées par des différences morales ou psychologiques générales) (VII 18-32). L'oikos est certes le lieu de manifestation de ces différences, mais il en est surtout la matrice, soit l'institution qui les rend significatives et opératoires. L'idée que la santé et la beauté de la femme d'Ischomaque - notamment la couleur de son teint (IX 11) - lui viendront de son engagement actif dans les tâches qui lui sont assignées (IX 9-13), tout comme le bénéfice qu'Ischomaque retire de ses activités économiques (aller aux champs, XI 14) pour sa santé (XI 14 ; XI 19), montrent que l'économie domestique est un discours et une pratique inscrivant les corps dans un ordre symbolique façonné par des valeurs : c'est par elle que la femelle acquiert un corps de femme, et le mâle un corps d'homme.

\subsection{L'eugénisme d'Aristote : les conditions biologiques de la production des corps des hommes libres}

Platon et Aristote, en revanche, font de la procréation l'un des buts principaux de l'union conjugale, car ils y voient l'un des ressorts essentiels de la perpétuation de l'oikos et de la cité. C'est toutefois sur la nature de ces propos eugénistes qu'ils se séparent, c'est-à-dire sur les facteurs pertinents de la production des corps les meilleurs, du moins les plus souhaitables dans le cadre de leurs propos. Commençons par Aristote. Un passage des Politiques est consacré à cette question (VII 16, 1334b30-1336a2), dans le cadre plus général d'une réflexion sur la fonction éducative du législateur, qui doit porter aussi bien sur le corps que sur l'âme (1334b25-26). C'est directement à propos du corps pour faire en sorte que « les enfants aient le meilleur corps possible» 
(1334b30) - que le législateur doit d'abord intervenir dans les unions : son objectif est de favoriser la naissance de corps possédant « une bonne

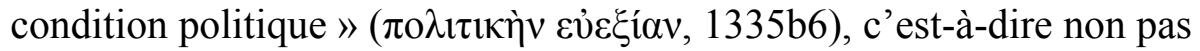
des corps d'athlètes mais des corps se prêtant aux activités des hommes

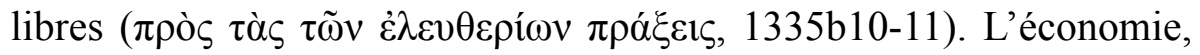
dans son versant humain, est directement pensée comme une entreprise de production d'un corps politique.

Deux questions guident Aristote pour déterminer l'action du législateur en ce sens : « quand et entre quelles sortes de gens doivent

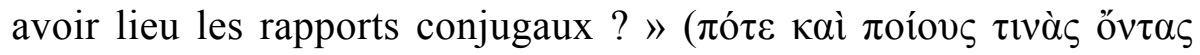

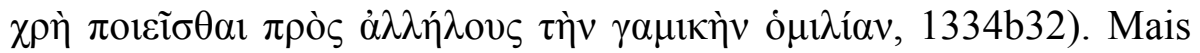
en réalité, la seconde question est traitée avec la première, au sens où l'âge des géniteurs exerce une influence sur leurs dispositions, morales et intellectuelles, influence qui peut se reporter sur leur progéniture. Traiter du « quand ? », c'est donc en même temps traiter du « de quelles sortes? ». Ces deux questions qui n'en sont qu'une concernent ce qu'on pourrait appeler le temps optimal de l'union, c'est-à-dire : 1) à partir de quel âge doivent débuter les unions, donc les mariages ?2) Quand est-il souhaitable que la procréation cesse?

1) Pour déterminer l'âge à partir duquel les relations peuvent commencer, Aristote mentionne plusieurs facteurs à prendre en compte : la durée de vie de l'homme et de la femme, la contemporanéité des âges à partir desquels leur fertilité baisse (pour éviter les mésententes que provoque, selon Aristote, leur non-coïncidence ${ }^{18}$ ), et la prise en compte des écarts optimaux entre l'âge des enfants et l'âge des parents, pour qu'il n'y ait ni trop ni trop peu de proximité dans ces relations mutuelles, la trop grande proximité en particulier étant « source de récriminations

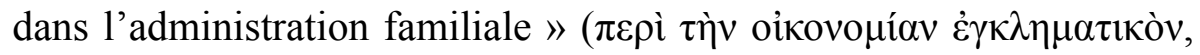
1335a3-4), sans doute parce qu'elle ne permet pas au père d'exercer convenablement sa fonction paternelle (1334b33-1335a6).

\footnotetext{
${ }^{18}$ Aristote n'explique pas les raisons de ces différends liés au décalage dans la procréation. Faut-il y lire la trace d'un risque pour l'accomplissement de la finalité de la nature - laisser un être semblable à soi ? - ou des considérations sociales, liées à la transmission du patrimoine par exemple?
} 
C'est le critère de l'âge de la cessation de la fertilité chez les deux sexes - 70 ans chez les hommes, 50 ans chez les femmes (1335b7-11) qui doit déterminer l'âge du début leur union, ce qui invite à écarter les unions trop précoces - aussi bien pour des raisons biologiques (les rejetons sont imparfaits : plus petits, plutôt femelles que mâles (1335b12-17), et cela affecte la croissance des pères) que morales (les jeunes filles qui ont des relations sexuelles jeunes en seraient rendues plus licencieuses ou débridées, $\grave{\alpha} \kappa o \lambda \alpha \sigma \tau o ́ \tau \varepsilon \rho \alpha 1,1335 b 24)$. Ces critères doivent conduire le législateur à déterminer l'âge minimal pour se marier vers 18 ans pour les filles et un peu moins de 37 ans pour les hommes (1335b28-30), car les corps sont alors au point optimal de leur épanouissement. Quant à connaître la période optimale de procréation dans l'année, Aristote renvoie à des savoirs plus spécialisés, ceux des puériculteurs et des médecins.

2) Quant à l'âge limite de la procréation, c'est-à-dire l'âge audelà duquel le corps du nouveau-né risque de ne plus avoir la «bonne disposition politique », Aristote en donne une idée approximative fondée

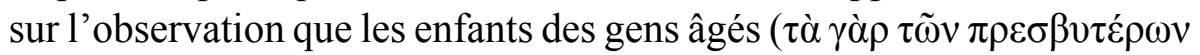

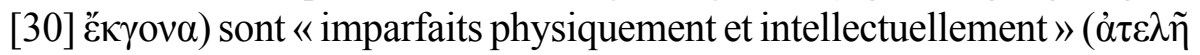

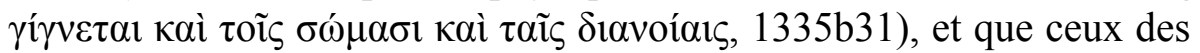

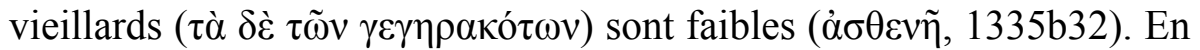
somme, après la cinquantaine, il faut être "déchargé [à $\varphi \varepsilon i ̃ \sigma \theta \alpha 1]$ de la fonction de donner le jour » (1335b37). Aristote ne dit pas comment le législateur doit s'y prendre pour y parvenir et n'évoque aucune interdiction concernant l'activité sexuelle à cet âge et au-delà. Les brèves lignes sur l'adultère qui terminent le passage précisent qu'il faut que « soit

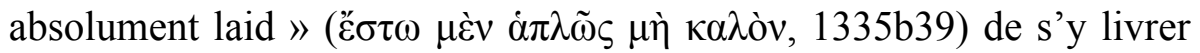

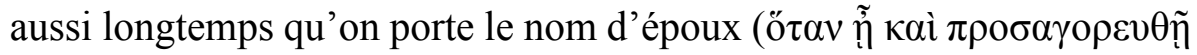

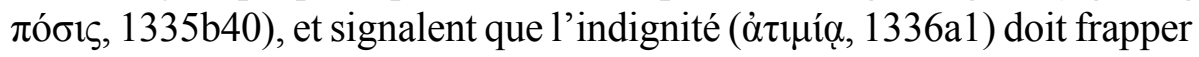
ceux qui s'y livrent alors qu'ils sont encore dans la période de procréation. S'agit il d'une seule et même faute, qui ferait coïncider le statut marital et la période de procréation ? Ou s'agit-il - ce qui semble plus vraisemblable étant donné que la relation conjugale déborde la période de la procréation - de deux sortes de punitions, l'une morale, au nom de la stabilité de la relation conjugale et de l'institution familiale, l'autre légale et civique, 
apparemment plus forte, peut-être parce qu'elle met en péril la linéarité de la succession et de l'héritage au sein de l'oikos?

Quoi qu'il en soit, qu'est-ce que ce corps civique qu'il s'agit de produire ? Défini par les activités dont il doit être capable une fois parvenu à maturité - celles de l'homme libre, c'est-à-dire à la fois celles du citoyen participant aux magistratures, celles de l'homme vertueux et éventuellement celles de l'homme tourné vers les activités théorétiques il prend sens dans le cadre plus général d'une politique des corps dont le maillon économique, qu'Aristote dépeint rapidement ici, est à l'évidence le plus déterminant. Aristote évoque en effet deux autres corps dans ce passage : celui des femmes enceintes, qui sont invitées à en " prendre

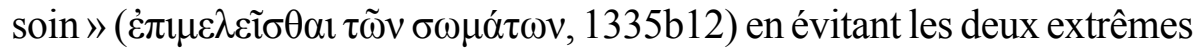
du laisser-aller et de la frugalité ; et le corps infirme ( $\pi \varepsilon \pi \eta \rho \omega \mu \varepsilon ́ v o v$, 1335b21) de certains nouveau-nés, qui doit être exposé (1335b19-21). Concernant ces derniers, Aristote ne précise pas quelles infirmités ou quel degré d'infirmité est visé, ou s'il s'agit d'une évocation indirecte des enfants de sexe féminin, dont on sait qu'il les présente comme des mâles

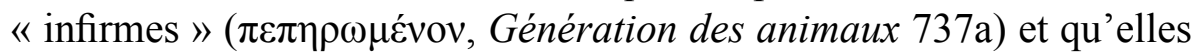
faisaient davantage l'objet de l'exposition que les enfants mâles. ${ }^{19}$

L'enjeu de l'union conjugale est donc bien, en tant que dimension fondamentale de l'économie domestique, de réaliser dans le corps l'articulation du biologique et du politique. L'économie domestique est, en ce sens, le lieu d'une « biopolitique ».

\subsection{L'eugénisme de Platon : les conditions éthiques de la procréation}

L'eugénisme comme pratique inhérente à l'économie domestique est présent aussi dans les Lois de Platon. Selon l'Athénien en effet, « il faut que la jeune mariée et le jeune marié considèrent qu'ils devront donner à la cité les enfants les plus beaux et les meilleurs possible » (VI 783d) : l'union conjugale, comme dimension de l'économie domestique, doit, comme chez Aristote, produire un corps et une âme politiques, ou aptes à le devenir (la « beauté » en question étant peut-être synonyme

${ }^{19}$ BRULÉ, 2009. 
du « sans infirmité » d'Aristote). Mais à la différence d'Aristote, c'est moins à la condition biologique générale du corps des parents que Platon s'intéresse pour que ce but soit atteint, qu'à leur disposition éthique, laquelle se répercute selon lui sur leur propre corps et, par l'intermédiaire de la procréation, sur l'âme et le corps de l'embryon. Aussi l'Athénien évoque-t-il les conditions ou les circonstances dans lesquelles doit avoir lieu la procréation, en distinguant le cas particulier de la procréation lors de la nuit de noces du cas général de la procréation au cours de la vie conjugale. Tout d'abord, c'est la conduite des parents, et en particulier du père, le soir des noces qui est déterminante : le risque de procréer ce soir-là est, sous l'effet du vin qui accompagne les festivités, de ne pas communiquer à l'embryon les qualités de consistance, stabilité, tranquillité qu'il doit avoir (VI 775c). En effet,

1'homme pris de vin donne et reçoit des chocs de toutes les manières, de violents transports agitant son corps et son âme. Celui qui est ivre est un donneur de semence mauvais et vacillant, de sorte que, selon toute probabilité, il engendrera des êtres mal équilibrés et mal venus, rien qui aille droit son chemin, pas davantage selon le corps que selon le caractère. (PLATON, Lois VI 775c-d).

L'Athénien étend ensuite son propos à la procréation qui n'a pas lieu le soir des noces mais dans la suite de la vie conjugale :

Aussi doit-on toute l'année, toute sa vie, et plus encore tout le temps que l'on procrée, être sur ses gardes et veiller à ne rien faire de son plein gré qui soit malsain ni rien qui ressortisse à la démesure ou à l'injustice. Car forcément l'être engendré en reçoit 1'empreinte dans son âme et dans son corps, et on donne fatalement le jour à des êtres qui ne vaudront rien à aucun égard. (PLATON, Lois VI 775d-e).

Il difficile de savoir si l'Athénien croit vraiment à une transmission biologique des dispositions éthiques, ou plus exactement, à l'impact psychique et physique sur les embryons des dérèglements 
éthiques de leurs géniteurs $;{ }^{20}$ ou s'il s'agit seulement d'une croyance destinée à asseoir dans l'esprit des citoyens la continuité éthique de la sphère économique ou domestique et de la sphère politique. Quoi qu'il en soit, les mesures qu'il prévoit pour que la procréation se réalise dans les meilleures conditions sont de nature éthique et politique : le jeune marié doit prêter attention à son épouse et à la procréation, et le couple recevra la visite d'inspectrices chargés de s'assurer, pendant les 10 ans assignés à la période de procréation à partir de l'âge du mariage (entre 16 et 20 ans pour les filles, 30 et 35 pour les garçons, VI 785b), qu'ils prennent cette tâche au sérieux (VI 783e-784a). Cette dimension particulière de l'économie domestique, entièrement subordonnée à une visée politique, n'a donc pas pour seul objectif de donner des corps à la cité, mais d'inscrire l'ordre de la cité à même le corps des individus. L'économie domestique, envisagée au niveau de l'union entre les époux, constitue de ce point de vue le point d'ancrage fondamental de la politique. S'unir - cela vaut pour Aristote, Xénophon, et de façon plus explicite encore pour Platon - c'est écrire la politique sur et dans les corps.

\section{Conclusion}

L'union conjugale est, on le voit, une dimension fondamentale de la réflexion philosophique classique concernant l'économie. Elle fait l'objet de débats et de controverses concernant ses principes, sa nature et sa finalité. Pièce centrale de l'édifice sociopolitique et, plus largement, de la relation de l'être humain au monde, elle est décisive parce qu'elle est le lieu et le principe de l'articulation entre le biologique et le politique, par quoi l'ordre de la cité s'inscrit dans le corps de ses membres et, par lui, dans leur âme. ${ }^{21}$

\footnotetext{
${ }^{20}$ On trouve une idée similaire à propos des rapports de justice et d'injustice entre les individus : République I 335c ; cf. Gorgias 476b.

21 Je remercie Jean-Baptiste Gourinat et Marwan Rashed de m'avoir accueilli au Centre Léon Robin où j'ai présenté une première version de ce texte sous la forme d'une communication, le 23 novembre 2018. Mes remerciements vont aussi à Olimar Flores-Júnior, María del Pilar Montoya et Paola Schirripa pour leur lecture et leurs précieux commentaires.
} 


\section{Références}

ARISTOTE. Économique. Texte établi par B. A. van Groningen et A. Wartelle, traduit et annoté par A. Wartelle. Paris : Les Belles Lettres, 1968. BRULÉ, P. L'exposition des enfants en Grèce antique : une forme d'infanticide. Enfances \& Psy, Paris, v. 44, n. 3, p. 19-28, 2009.

COX CHERYL, A. Household Interests. Property, Marriage Strategies, and Family Dynamics in Ancient Athens. Princeton : Princeton University Press, 1998.

DAMET, A. ; MOREAU, Ph. Famille et société dans le monde grec et en Italie du Ve s. av J.-C. au IIe s. av. J.-C. Paris : Armand Colin, 2017.

DESCAT, R. Aux origines de l' oikonomia grecque. Quaderni Urbinati di Cultura Classica, Roma, N.S. v. 28, n. 1, p. 103-119, 1988.

FOUCAULT, M. L'Histoire de la sexualité. v. 2 : L'usage des plaisirs. Paris : Gallimard. 1984.

HELMER, É. Réévaluer la réflexion grecque sur l'économie: de la science économique à la philosophie de l'économie. Mètis, Paris, N.S. v. 14, p. 187-205, 2016.

HELMER, É. Le remodelage politique de l'oikos dans la République: de la famille au modèle familial, de l'économie domestique à l'économie politique. Plato - The Internet Journal of the International Plato Society, n. 11, s.p. 2011. URL: <http://gramata.univ-paris1.fr/Plato/spip. php?article98> .

KING, C. Musonius Rufus : Lectures and Sayings. Translated with an Introduction by Cynthia King ; edited with a Preface by William B. Irvine. [USA] : CreateSpace, 2011

LESHEM, D. The Ancient art of Economics. The European Journal of the History of Economic Thought, v. 21, n. 2, p. 201-229, 2012.

NATALI, C. Oikonomia in Hellenistic Political Thought. In : LAKS, A.; SCHOFIELD, M. (Ed.). Justice and Generosity: Studies in Hellenistic Social and Political Philosophy Proceedings of the Sixth Symposium Hellenisticum. Cambridge : Cambridge University Press, 1995. p. 95-128. 
POMEROY, S. Goddesses, Whores, Wives and Slaves. Women in Classical Antiquity. New York : Schocken Books, 1995 [1975].

SIMPSON, P. A Philosophical Commentary on the Politics of Aristotle. Chapel Hill/London : University of North Carolina Press, 1998.

Recebido em: 20 de setembro de 2018. Aprovado em: 28 de novembro de 2018. 